\title{
Simple device for pediatric and adult humeral supracondylar fracture
}

\section{Introduction}

Pediatric supracondylar fracture accounts for $60 \%-80 \%$ of pediatric elbow fracture being the highest rate of complications of any pediatric fractures. Prompt reduction and stabilization reduce the incidence of complication.

Closed reduction and per-cutaneous pinning is the gold standard treatment of choice. It is considered an emergency, needs quick treatment especially at night Handled by single on call junior orthopedic surgeon in time with no senior expert surgeon available, it will be difficult to reduce and maintain without complication, due to anatomic consideration.

A $5 \$$, simply designed device , made by radiolucent PVC water pipes, will be able to allow easy reduction and maintains it till preoperative sterility and adjusting $\mathrm{x}$ ray position., ${ }^{1,2}$

Easily operated by one junior on call orthopedic, it will allow the surgeon to operate in a comfortable sitting position adding to the accuracy and precision of the insertion of the pins. Less added trauma and manipulation.

\section{Methods}

Under G.A, the device will be fixed to the table rail. Close Reduction will be done then the forearm will be fixed temporary to the horizontal bar of the device by a skin traction rope. After sterilizing and draping of both the limb and device, we will put the tip of the medial pin just anterior to the proximal $1 / 2$ of the medial epicondyle, then; withdraw slowly medially until touching the medial ridge of the upper and anterior $1 / 2$ of the medial epicondyle. Angulate the pin 40 laterally, 20 posteriorly in the line of the medullary cavity, protect the ulnar nerve by the left thumb then push the tip by prying with no power. After that, insert slowly $1 \mathrm{~cm}$ by the driver and verify by $\mathrm{x}$ ray both AP and LAT, if ok continue till reaching the lateral cortex. Direct the Lateral pin in $20^{\prime}$ medially and posteriorly $40^{\circ}$, by prying insert the tip of the pin without power then slowly drive the pin about $1 \mathrm{~cm}$. Confirm by image intensifier, if the direction is acceptable, continue until reaching the other posterior cortex until the normal resistance release .Confirm by x ray and don't use one go technique. Now, we use two pins laterally and one pin medially. ${ }^{2-5}$

\section{Results}

We used this technique, with the help of the device, for pediatric supracondylar fracture for more than 15 years, with a very good outcome in more a hundred cases. Nowadays, we started to use it for adult complex lower end humerus fracture in some groups of polytrauma unstable patients, particularly those with chest injuries, head injuries, and those with mangled extremities as a quick minimal invasive surgery for temporarily stabilizing and causing no second immunological hit to these unstable critical multiple injury patients. As damage control orthopedic. It was used in few cases, with a promising outcome. Two adolescents, one female and one male, 13 and 14 years of age respectively, with the first case being an open contaminated fracture due to late referral. Both were isolated fractures (Figures 1-7).
Volume 10 Issue 3 - 2018

\author{
Safaa-Eldin Elsayed Abaza \\ Orthopedic consultant, FRCS GLASGOW, UAE
}

\begin{abstract}
Correspondence: Safaa-Eldin Elsayed Abaza, Orthopedic consultant, FRCS GLASGOW, Gulf Diagnostic Center Hospital Abu Dhabi, UAE, Tel +97/504I46390,
\end{abstract}

Email safaa.abaza@yahoo.com

Received: May 22, 2018| Published: June 21, 2018

Another case of one woman 49 year of age with isolated fracture with osteoporosis .Five cases as DCO with multiple injury patients, all are male with massive chest, head and abdominal injuries and other co morbidity and fractures used as a temporary fixation during first day handling for another emergency surgery. ${ }^{6,7}$

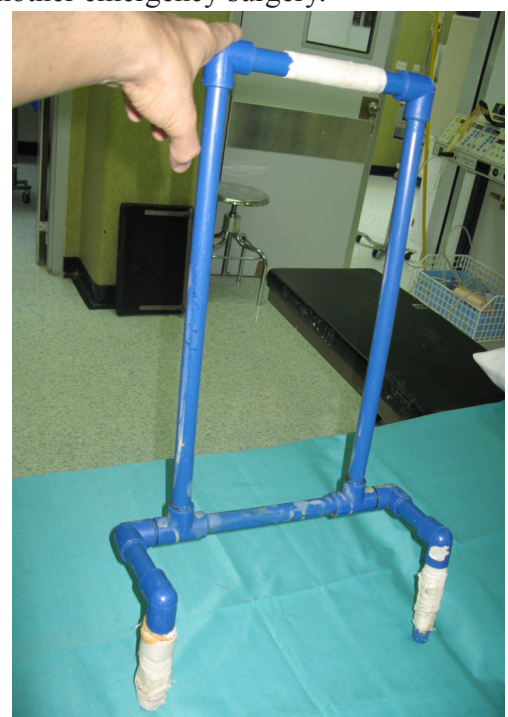

Figure I

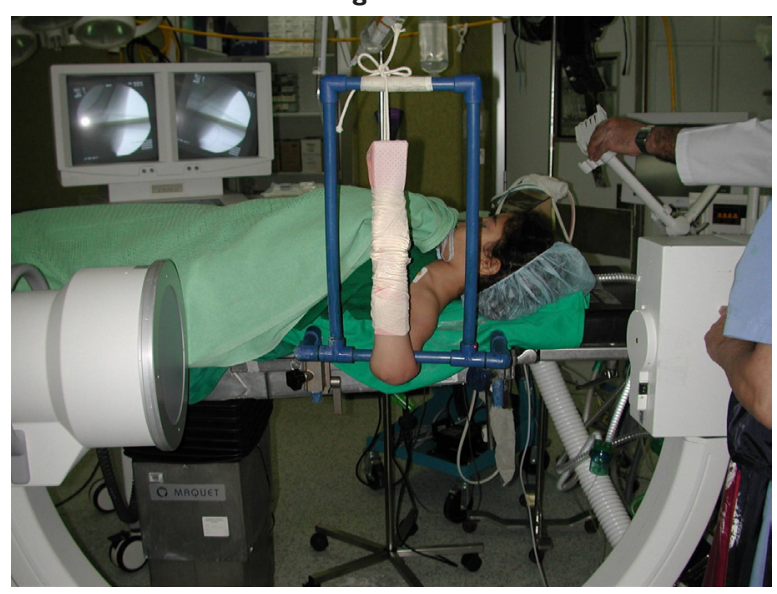

Figure 2 


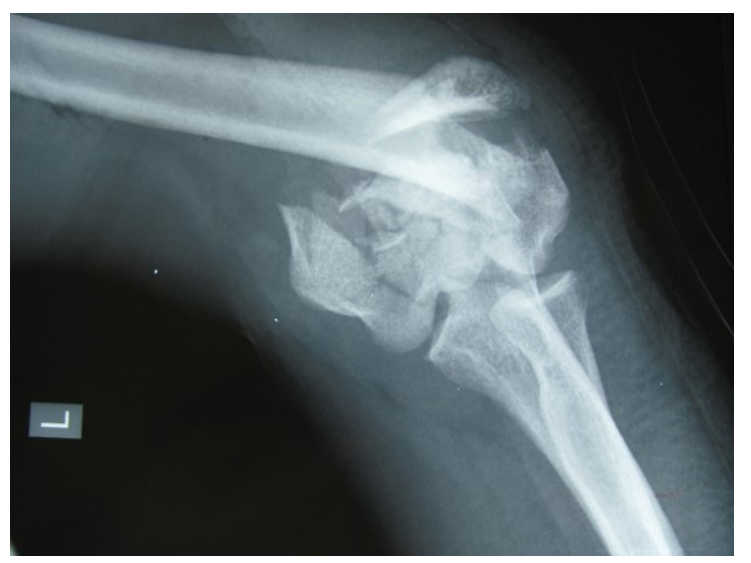

Figure 3

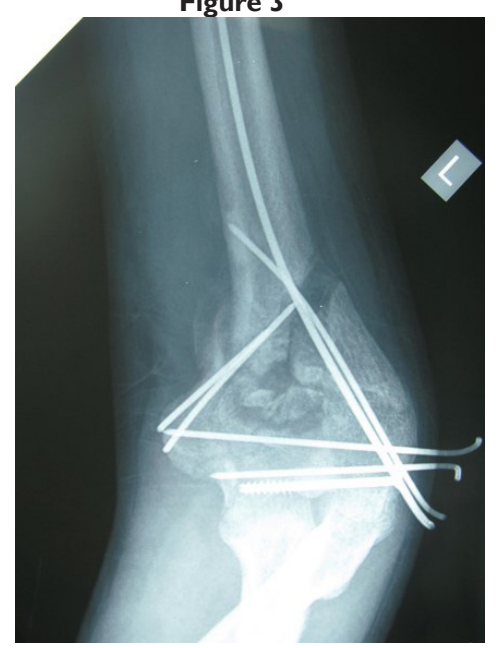

Figure 5

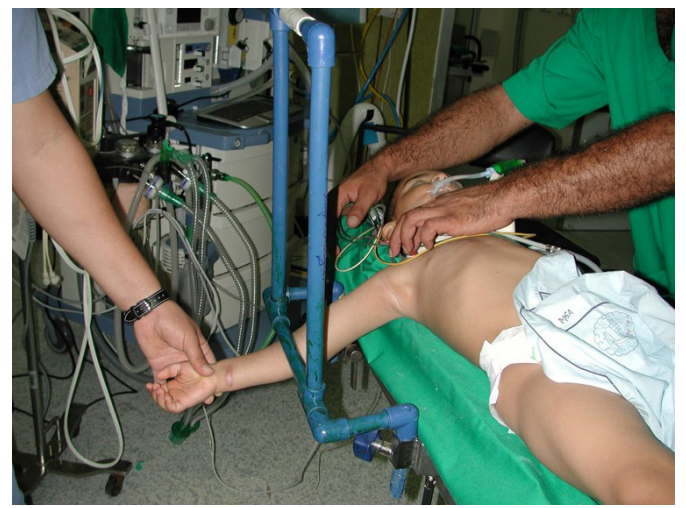

Figure 7

\section{Discussion and conclusion}

Pediatric supracondylar fracture is a common fracture with the close reduction and per-cutaneous pinning being difficult to reduce and maintain by average first on call health care providers. We were able to assemble and utilize this simple and cheap traction device to ease our handling and manipulating of such a fracture. Making treatment of this fracture very simple. We were able to utilize it in sever adult complex lower end humeral fracture giving promising results.

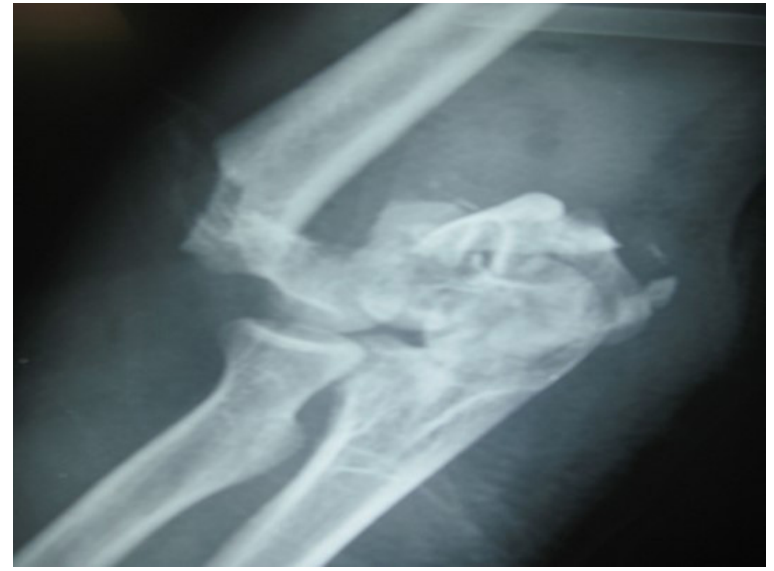

Figure 4

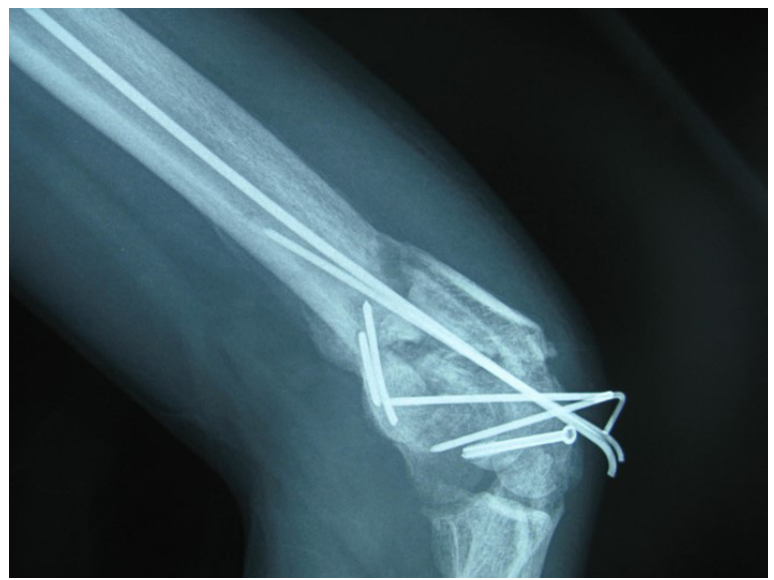

Figure 6

\section{Acknowledgements}

None.

\section{Conflict of interest}

The author declares no conflict of interest.

\section{References}

I. Pollock JW, Faber KJ, Athwal GS. Distal humerus fractures. Orthop Clin North Am. 2008;39(2): 187-200.

2. Wong AS, Baratz ME. Elbow fractures: distal humerus. J Hand Surg. 2009;34(I): 176-90.

3. Reising K, Schmal H, Kohr M, et al. Surgical Treatment of Supracondylar Humerus Fractures in Children. Acta Chir Orthop Traumatol Cech. 201 I;78(6):519-23.

4. Leitch KK, Kay RM, Femino JD, et al. Treatment of multidirectionally unstable supracondylar humeral fractures in children. A modified Gartland type-IV fracture.J Bone Joint Surg Am. 2006;88(5):980-5

5. British orthopaedic association standards for trauma. BOAST II: supracondylar fractures of the humerus in children. 2014.

6. Lim KB, Lim CT,Tawng DK. Supracondylar humeral fractures in children: beware the medial spike. Bone Joint J. 2013;95-B(9): 1290-4.

7. Kosuge D, Barry M. Changing trends in the management of children's fractures. Bone Joint J. 2015;97-B(4):442-8. 